\title{
BMJ Open Screening test accuracy to improve detection of precancerous lesions of the cervix in women living with HIV: a study protocol
}

Katayoun Taghavi (D) , ${ }^{1,2}$ Misinzo Moono, ${ }^{3}$ Mulindi Mwanahamuntu, ${ }^{4,5}$ Partha Basu, ${ }^{6}$ Andreas Limacher, ${ }^{7}$ Taniya Tembo, ${ }^{3}$ Herbert Kapesa, ${ }^{3}$ Kalongo Hamusonde, ${ }^{3}$ Serra Asangbeh, ${ }^{1,2}$ Raphael Sznitman, ${ }^{8}$ Nicola Low (D) , , Albert Manasyan,, Julia Bohlius ${ }^{1}$

To cite: Taghavi K, Moono M, Mwanahamuntu $\mathrm{M}$, et al. Screening test accuracy to improve detection of precancerous lesions of the cervix in women living with HIV: a study protocol. BMJ Open 2020;10:e037955. doi:10.1136/ bmjopen-2020-037955

- Prepublication history and additional material for this paper are available online. To view these files, please visit the journal online (http://dx.doi. org/10.1136/bmjopen-2020037955).

Received 22 February 2020 Revised 07 November 2020 Accepted 17 November 2020

A) Check for updates

(c) Author(s) (or their employer(s)) 2020. Re-use permitted under CC BY-NC. No commercial re-use. See rights and permissions. Published by BMJ.

For numbered affiliations see end of article.

\section{Correspondence to} Dr Katayoun Taghavi; katayoun.taghavi@ispm.unibe. ch

\section{ABSTRACT}

Introduction The simplest and cheapest method for cervical cancer screening is visual inspection after application of acetic acid (VIA). However, this method has limitations for correctly identifying precancerous cervical lesions (sensitivity) and women free from these lesions (specificity). We will assess alternative screening methods that could improve sensitivity and specificity in women living with humanimmunodeficiency virus (WLHIV) in Southern Africa.

Methods and analysis We will conduct a paired, prospective, screening test accuracy study among consecutive, eligible women aged 18-65 years receiving treatment for HIV/AIDS at Kanyama Hospital, Lusaka, Zambia. We will assess a portable magnification device (Gynocular, Gynius Plus AB, Sweden) based on the Swede score assessment of the cervix, test for high-risk subtypes of human papillomavirus (HR-HPV, GeneXpert, Cepheid, USA) and VIA. All study participants will receive all three tests and the reference standard at baseline and at six-month follow-up. The reference standard is histological assessment of two to four biopsies of the transformation zone. The primary histological endpoint is cervical intraepithelial neoplasia grade two and above (CIN2+). Women who are VIA-positive or have histologically confirmed CIN2+ lesions will be treated as per national guidelines. We plan to enrol 450 women. Primary outcome measures for test accuracy include sensitivity and specificity of each stand-alone test. In the secondary analyses, we will evaluate the combination of tests. Pre-planned additional studies include use of cervigrams to test an automated visual assessment tool using image pattern recognition, cost-analysis and associations with trichomoniasis.

Ethics and dissemination Ethical approval was obtained from the University of Zambia Biomedical Research Ethics Committee, Zambian National Health Regulatory Authority, Zambia Medicines Regulatory Authority, Swissethics and the International Agency for Research on Cancer Ethics Committee. Results of the study will be submitted for publication in a peer-reviewed journal.

Trial registration number NCT03931083; Pre-results.
Strengths and limitations of this study

- The study design allows evaluation of screening test accuracy of stand-alone and combinations of tests with adequate precision.

- This is the first study to assess the test accuracy of the Gynocular and validation of the Swede score assessment of the cervix in women living with human immunodeficiencyvirus (WLHIV).

- All screened women will receive the reference standard of histological assessment at both baseline and follow-up, thereby reducing verification and misclassification bias.

- We will test an automated visual assessment tool, using a deep-learning algorithm in WLHIV.

- We limited the number of screening tests to be evaluated and excluded Papanicolaou testing, testing for DNA methylation, and human papillomavirus oncoproteins $E 6$ and $E 7$, as these were less suitable to implement in our study setting.

\section{INTRODUCTION}

The risk of invasive cervical cancer is higher for women living with humanimmunodeficiency virus (WLHIV) than for HIV-uninfected women. ${ }^{1-4}$ Cervical cancer remains the leading cause of death from cancer among women in Zambia, despite the introduction of an integrated service for HIV care and cervical screening in $2006 .^{1}$ In 2020, human papillomavirus (HPV) vaccination was rolled out for adolescent girls in Zambia, but the effects on reducing the burden of cervical cancer will not be seen for at least 10 years. ${ }^{5}$ Until then, secondary screening strategies are essential. Existing screening strategies in Southern Africa, so far, have not reduced the high burden of disease. ${ }^{15}$ The simplest and cheapest method for cervical cancer screening is visual inspection of the uterine 


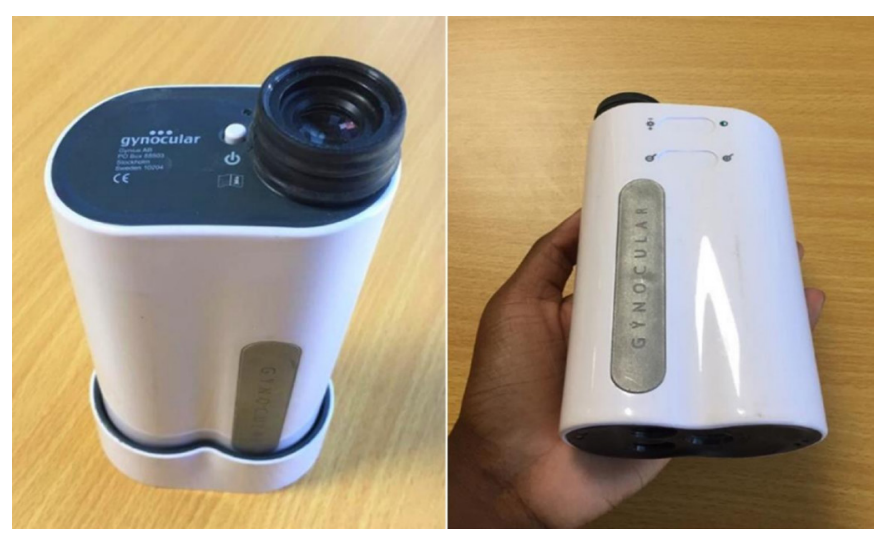

Figure 1 The Gynocular (Gynocular, Gynius Plus AB, Sweden). This photograph was taken at the Centre for Infectious Disease Research in Zambia headquarters on the 27th of April 2020 for the purposes of this manuscript.

cervix after application of $3 \%-5 \%$ acetic acid (VIA), and this method is employed widely in low- and middleincome countries (LMICs). When used as a stand-alone test, VIA has variable accuracy and high subjectivity. ${ }^{6}$ Studies in sub-Saharan Africa have reported large variations in sensitivity of VIA when compared with histological assessment: $25 \%$ (95\% CI 7\% to 59\%) in Cameroon, ${ }^{7}$ $48 \%(95 \%$ CI $30 \%$ to $67 \%)$ in $\mathrm{Zambia}^{8}$ and $86.6 \%(95 \%$ CI $81.1 \%$ to $91.6 \%$ ) in Kenya. ${ }^{9}$ The test accuracy of VIA is low when compared with the tests in screening pathways of high-income countries, which include Papanicolaou smear, testing for high-risk HPV (HR-HPV testing) and colposcopy, especially among WLHIV. ${ }^{6-12}$ Papanicolaou testing is hard to implement and scale-up in many countries, including Zambia, owing to shortages of pathologists and high loss to follow-up. ${ }^{4}$

The World HealthOrganization (WHO) advocates for urgent investigation into the best methods of cervical cancer screening for WLHIV in LMICs, ${ }^{12}$ but a comprehensive evaluation of alternatives has not yet been performed. In sub-Saharan Africa, screening for precancerous lesions of the cervix using HR-HPV testing has a sensitivity of $88.3 \%$ (95\% CI $73.1 \%$ to $95.5 \%$ ) and specificity of $73.9 \%$ (95\% CI $50.7 \%$ to $88.7 \%$ ). ${ }^{13}$ The WHO call for the elimination of cervical cancer has led to huge efforts to improve access to point-of-care HR-HPV testing in countries where this was previously too expensive to implement. ${ }^{6}{ }^{14}$ It may soon be possible to roll out widespread HR-HPV testing in many LMICs. However, the high prevalence of HR-HPV infection in WLHIV ${ }^{15}$ means that a screening strategy using HR-HPV alone may leave many women in need of treatment. This study provides an opportunity to assess the test accuracy of HR-HPV as a stand-alone test, and in combination with other tests, which might aid in treatment decisions.

We will investigate screening methods that could improve sensitivity and specificity for the detection of precancerous cervical lesions in WLHIV and could be scaled up in Zambia. We consider three strategies: Gynocular (Gynocular, Gynius Plus AB, Sweden), HR-HPV
(GeneXpert, Cepheid, USA) and VIA, that adhere to the 'screen and treat' principle. This is essential to ensure that screening is adequately linked to treatment and to minimise loss to follow-up. In addition to VIA and HR-HPV testing, we will assess the Gynocular mobile magnification device and Swede score of severity of cervical lesions. The Gynocular (figure 1) is battery-operated, allows inspection with a green light, has two brightness settings for the white light and has three magnification settings $(5 \times, 8 \times, 12 \times)$, which allows magnified examination of the cervix, exceeding what is possible from low-magnification devices. Five published studies have examined the potential applications of the Gynocular in a general population. ${ }^{16-20}$ In clinical settings, the Gynocular is used with a validated scoring system for assessment of the cervix, the Swede score (the Swedish score), a tool to make colposcopic diagnosis less subjective. ${ }^{21}$ This study includes the first assessment of the Gynocular in WLHIV. The methods of the present study also allow the incorporation of substudies, including: (1) investigation of the accuracy of an automated visual assessment tool, using a deep-learning method for image recognition, outside of the clinical setting, which has shown potential in a study published in $2019^{22}$; (2) a cost-analysis; and (3) a study of associations between Trichomonas vaginalis and vaginal and menstrual hygiene practices. ${ }^{23} 24$ The methods and statistical analysis plans for these studies will be described separately.

In this paper, we present the protocol for a clinical study to estimate test accuracy of Gynocular, HR-HPV and VIA in a stand-alone capacity, and in combination, in WLHIV in Lusaka, Zambia.

The primary objective is to:

1. Estimate the sensitivity and specificity of the Gynocular, HR-HPV and VIA when used as stand-alone tests to detect cervical intraepithelial neoplasia, grade two and above (CIN2+) among WLHIV.

The secondary objectives are to:

1. Determine other measures of test accuracy of the Gynocular, HR-HPV testing and VIA.

2. Determine test accuracy for combinations of screening tests, that is, HR-HPV followed by Gynocular or VIA.

3. Investigate effects of patient characteristics on test accuracy.

4. Determine optimal cut-offs for Swede score assessment of cervical lesions to identify CIN2+ lesions among WLHIV.

\section{METHODS}

\section{Study design}

This study received ethical approval and is reported in accordance with the 2015 'Standards for Reporting Diagnostic accuracy studies' (STARD) guidelines. We present a single site, paired, prospective, screening test accuracy study in WLHIV in Lusaka, Zambia. All data collection is performed prospectively. Study data are recorded using paper and electronic Case Report Forms 
(CRFs). A trained data associate enters data from paper CRFs into a password-protected electronic database (Research Electronic Data Capture, Vanderbilt University, Nashville, USA) once a week. This secure web application managing online databases is monitored with the support of the Clinical Trials Unit at the University of Bern. All paper form entries and electronic form entries are verified.

\section{Participants}

The study population includes women enrolled in the antiretroviral therapy (ART) programme at Kanyama hospital in Lusaka, Zambia. In addition to ART services, the hospital has provided cervical screening since October 2006. The clinic has a well-established, VIA-based 'screen and treat' approach to testing for precancerous cervical lesions. The main referral site for the study is the University Teaching Hospital, the tertiary level hospital in Lusaka, Zambia.

Patient enrolment occurs during the working hours of the ART clinic. A study research assistant gives a prescreening sensitisation talk to groups of women during their HIV clinic visits. The talk includes information about the study requirements, as well as the benefits and risks of being involved. Women who are interested in participating then present themselves to the cervical screening clinic check-in desk, which is in a neighbouring building on the same hospital site. A study assistant determines eligibility. The study assistant explains the study in more detail and obtains written consent from eligible women in a private room. The consent form is available in two local languages (Nyanja and Bemba) and has been back translated into English to ensure accuracy. For illiterate study participants, a literate impartial witness is present during the entire consent process to ensure that all the relevant information has been provided and that the participant gives consent for participation voluntarily. These participants indicate their consent by placing their thumbprint on the consent form. Patients who do not wish to participate in this study continue to receive cervical cancer screening care and treatment according to the local standard of care. A maximum of four consecutive participants per day can be enrolled during clinic hours.

The inclusion criteria are:

1. Women attending the ART programme, with HIV infection status confirmed from medical records.

2. Living within Kanyama district and intending to stay in the area for the next six months.

3. Age between 18 and 65 years.

4. Capacity and willingness to give consent.

5. Willingness to undergo a pelvic examination and cervical cancer screening.

6. Ever having had sexual intercourse.

7. Agreement to return for a follow-up appointment in 6 months.

The exclusion criteria are:
1. A history of cervical cancer or hysterectomy where the cervix was also removed (previous treatment for precancerous lesions is permitted).

2. Pregnant or plans to become pregnant within six months of enrolment.

3. Having received vaccination against HPV.

To reduce the risk of selection bias, we do not exclude menstruating women but if blood obscures the view of the cervix through the speculum, we ask them to return for the examination after their menses. Enrolment started in May 2019. We plan to continue recruitment and follow-up until January 2021, but may need to amend these dates in response to the ongoing COVID-19 pandemic.

\section{Test methods and study procedures}

All consenting study participants complete a nurseadministered questionnaire by pen and paper, and undergo baseline testing for HIV RNA viral load, CD4 cell count and T. vaginalis. They also undergo all screening tests: HR-HPV testing, VIA screening and Gynocular assessment, in that sequence, during their first visit (figure 2). Two nurses and one research assistant are involved in data and specimen collection, and they perform their procedures in separate rooms. They record their results on separate report forms and are instructed not to communicate results together. The participants are also instructed not to communicate the findings of one nurse to another.

A clinic nurse first reviews the participants. She performs the first gynaecological examination and takes the specimen for HR-HPV testing, followed by the specimen for $T$. vaginalis testing, and then performs a VIA examination. The HR-HPV testing is done using a single-use cervical cytobrush provided by GeneXpert. The cytobrush specimen is placed into ThinPrep PreservCyt (Cepheid, Sunnyvale, California, USA) immediately after collection. The $T$. vaginalis test is taken using a cotton-tipped swab provided by GeneXpert. These samples are passed on to a research assistant, before commencement of the VIA examination. The specimens are processed at the same time by the GeneXpert machine (Cepheid, Sunnyvale, California, USA), according to the manufacturer's instructions. The machine is located in the cervical cancer screening clinic. HPV testing output is then categorised into negative, positive HPV 16, positive HPV 18 or 45, or positive for other HR-HPV, including the following HPV subtypes: 11, 31, 33, 35, 39, 51, 52, 56, 58, 59, 66 and 68 .

VIA is carried out using the methodology described by the International Agency for Research on Cancer (IARC).$^{24}$ This is summarised as follows: insertion of a speculum, followed by visualisation of the vagina, vulva and cervix; assessment with the naked eye after application of normal saline and further assessment after application of 5\% acetic acid for one minute. The nurse records the findings as normal, abnormal or suspicious of cancer. Local guidelines instruct VIA nurses to categorise indeterminate findings as abnormal. 


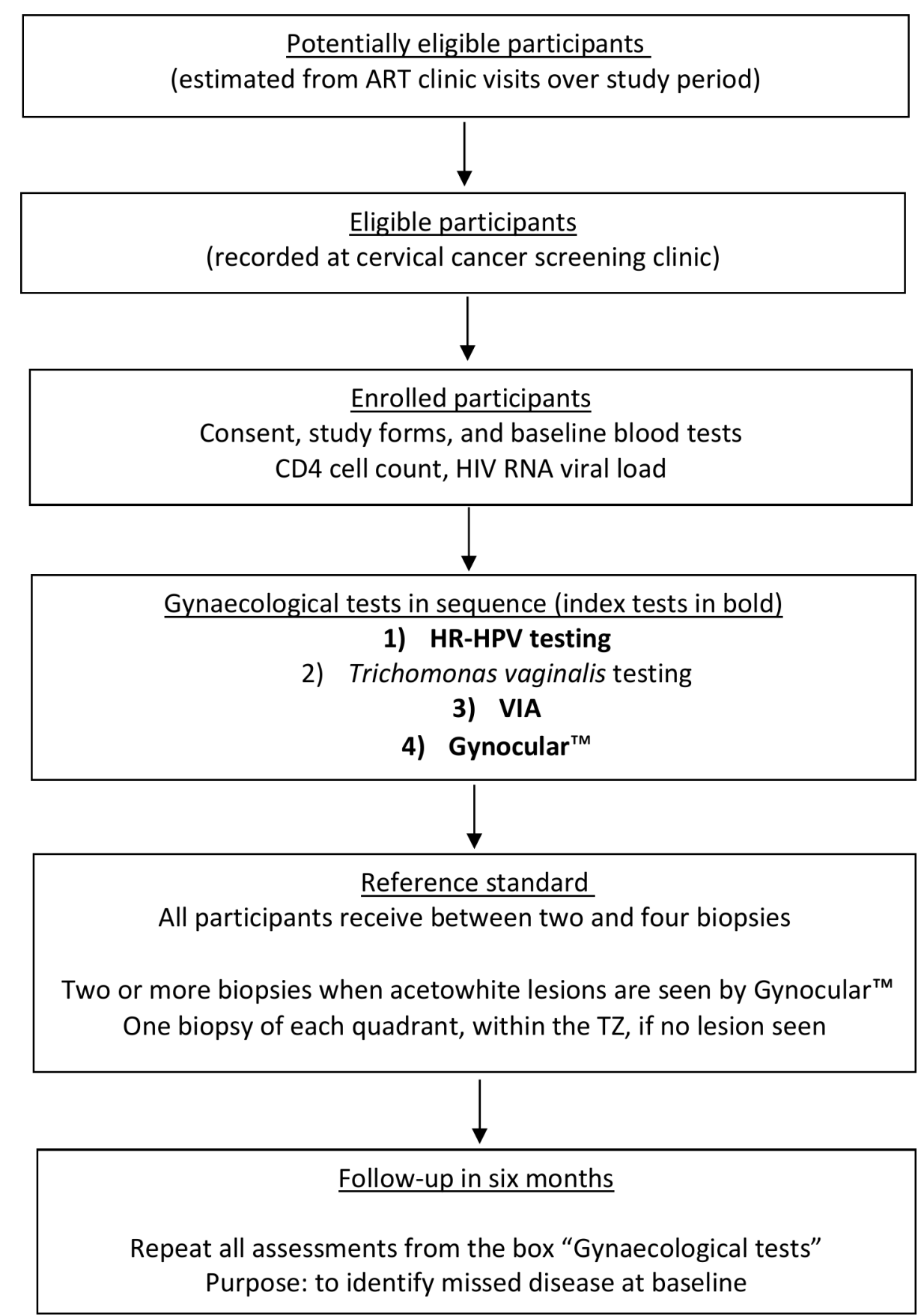

Figure 2 Flow of participants through the study*. VIA-positive women and those who have CIN2+ on histopathology will be treated as per national guidelines. Numbers of participants at each step will be reported. Loss to follow-up and missing data will also be reported. 'This flow diagram is in accordance with the 2015 'Standards for Reporting Diagnostic accuracy studies'. ART, antiretroviral therapy; CD4, cluster of differentiation 4; CIN2+, cervical intraepithelial neoplasia grade two and above; HRHPV, high-risk human papillomavirus; TZ, transformation zone of the cervix; VIA, visual inspection of the uterine cervix after application of $3 \%-5 \%$ acetic.

A different nurse performs the Gynocular examination, after VIA, in a different examination room, following the steps for colposcopy as described in the IARC colposcopy manual. ${ }^{24}$ These steps include insertion of a speculum followed by visualisation of the vagina, vulva and cervix, assessment of the cervix at low and high magnification $(>6 \times)$ after application of normal saline, examination of cervical vessel patterns using the red-free mode (or green filter), application of $5 \%$ acetic acid for one minute, and finally assessment following application with Lugol's iodine. The findings of the examination are documented using the Swede score to standardise the assessment of cervical lesions. ${ }^{2025}$ The Swede score uses five domains (vessels, margins or surface, acetic acid uptake, iodine staining and lesion size). Each domain is scored between zero and two, based on the severity of the findings, and summed to a total score between zero (best) and ten (worst). ${ }^{26}$ The test positivity cut-offs 
are not predefined in WLHIV and will be determined by our results. We did not train nurses to use endocervical forceps or perform endocervical curettage in the presence of a type 3 transformation zone, since the guidelines of the Zambian Ministry of Health do not allow nurses to perform these procedures. Women who are considered ineligible for treatment due to type 2 or type 3 transformation zone will be referred to a local gynaecologist for management.

The second study nurse takes cervical images using the smartphone attached to Gynocular at each stage of the examination. The images are stored electronically on a hard-drive and a secure server at Centre for Infectious Disease Research in Zambia (CIDRZ) headquarters. In a separate study, we will use coded images linked to anonymised clinical data obtained in the study, to test an automated visual assessment tool using deep-learning methods for image pattern recognition for the detection of CIN2+ lesions of the cervix.

The reference standard is the histological assessment of cervical tissue biopsies. All participants receive between two and four biopsies. At least two biopsies are obtained from women who have visible lesions. If no lesion is seen, four biopsies are obtained from clock-face positions of 12, 3, 6 and 9 o'clock within the transformation zone. All biopsies are taken perpendicular to the epithelium, are deep enough to sample the entire epithelium along with a small amount of stroma and are placed in formalin. To ensure standardisation, a study nurse received training in colposcopy and biopsy taking from a gynaecologist at IARC and a senior local gynaecologist.

All biopsies are sent for processing and examination at a laboratory in South Africa. An expert gynaecological pathologist, blinded to the visual assessment, will examine the biopsies. Slides are classified using the cervical intraepithelial neoplasia (CIN) classification system. CIN1 affects only the lower third of the epithelium (mild dysplasia), CIN2 involves two-thirds of the epithelium and CIN3 involves the full thickness (severe dysplasia and carcinoma in situ). These findings are then dichotomised into low-grade and high-grade squamous intraepithelial lesions (HSIL), by the Lower Anogenital Squamous Terminology definitions. All histology with CIN2 that stained diffusely positive for p16 is considered as HSIL, and all patients with CIN3 are considered as HSIL. Management decisions during the study will be based on the histological assessments done at this laboratory. At the conclusion of the study, an independent pathologist will verify all histopathology results, and we will use a consensus assessment for academic purposes in our analysis of outcomes.

The primary histological endpoint for our study is CIN2+. However, we will also determine the histological endpoint of HISL based on p16. To ascertain the presence of true high-grade cervical disease, we will rely on biopsy findings at baseline and 6-month follow-up. Disease detected at 6 months will be considered a missed case rather than a new case, because CIN progression is slow it is unlikely that a woman developed a new case in so short a time.

Women with VIA-positive findings are eligible for ablative treatment at their baseline visit, as per national guidelines. Treatment by cryotherapy or thermoablation is administered if the lesion fulfils the following criteria: boundaries are fully visible, covers less than $75 \%$ of the ectocervix, does not extend into the endocervical canal or the vagina, and is fully covered using the cryotherapy tip being used. ${ }^{25}$ VIA-positive women who are not eligible for immediate ablative treatment, and women who are assessed to have lesions suspicious of cancer, are referred immediately to the University Teaching Hospital or another nearby facility which offers the required treatment. All women with CIN2+ on histopathology and missed treatment at baseline (VIA-negative findings) will be offered treatment as per national guidelines.

The clinical pathway allows blinding of index tests and the reference standard for the clinical team performing tests. The clinic nurse performs VIA assessment and baseline tests first in a designated examination room. The participant then presents to a different examination room, where the second study nurse repeats the speculum examination and performs the Gynocular examination. To preserve blinding of the study nurse to VIA findings, the clinic nurse treats women with VIA-positive findings amenable to cryotherapy after completion of all other study procedures. She instructs the participant not to communicate this plan to the study nurse performing the Gynocular examination. Women with positive biopsy results receive treatment at a planned follow-up visit ('visit 2'). All women are followed up at 6 months ('visit 3'), during which the study participants undergo the same tests (HR-HPV, VIA, Gynocular and a biopsy).

Different assessors independently perform VIA (clinic nurse), HR-HPV testing (first study nurse) and Gynocular (second study nurse) examinations, and record results on separate data collection forms. GeneXpert HR-HPV testing follows the physical examinations; the result cannot influence the VIA nurse or Gynocular nurse assessments as it is only available after all visual examinations are complete. Biopsy results are known only after the completion of VIA, HR-HPV and Gynocular tests.

\section{Sample size calculation and analysis}

This screening test accuracy study requires 350 participants to estimate the sensitivity and specificity of Gynocular, HR-HPV and VIA for CIN2+ lesions with the precision detailed in table 1 . Screening accuracy measures will be estimated with $95 \%$ Wilson confidence intervals with no formal hypothesis testing between modalities.

We will enrol 450 women to obtain data from at least 350 patients for statistical analyses. We expect the prevalence of CIN2+ in WLHIV in Zambia to be 16\%-20\%. 82728 We expect disease prevalence to be lower than in previous years. Increases in the number of women receiving ART, and commencing treatment at higher $\mathrm{CD} 4$ cell counts, ${ }^{29}$ may lead to a decline in HPV prevalence. ${ }^{30}$ Higher rates 


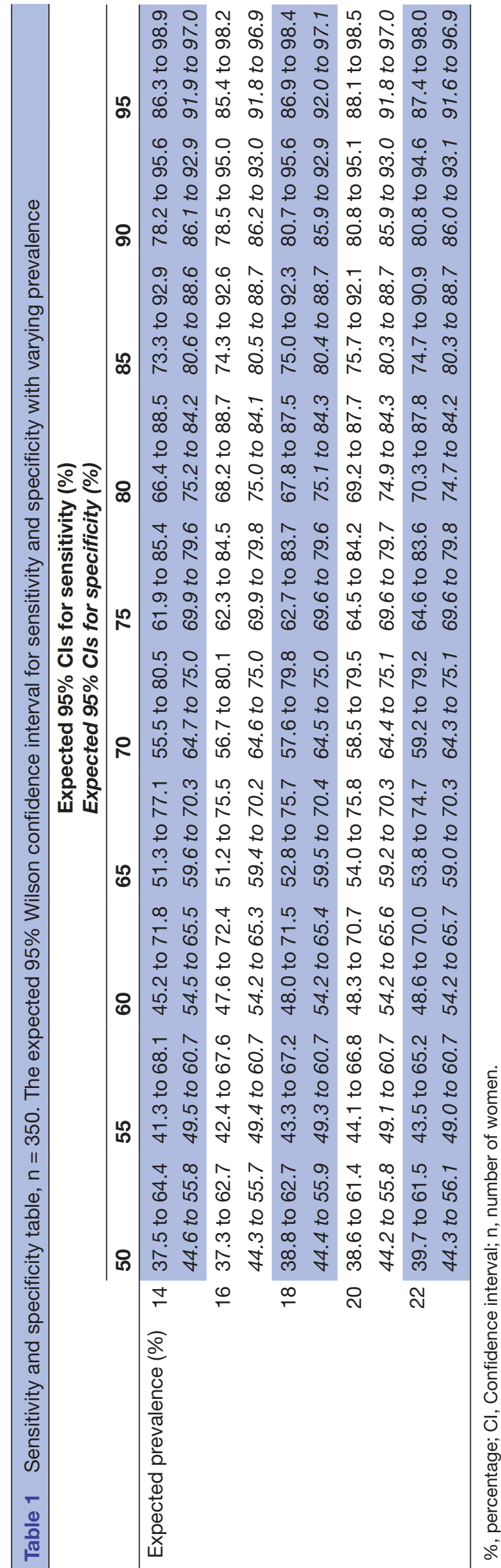

of voluntary male circumcision in male partners may also contribute to lower levels of HPV infection. ${ }^{30}$ We also estimate that there might be up to $10 \%$ loss to follow-up and up to $10 \%$ of tests that are not analysable or interpretable. We have implemented rigorous data collection methods to avoid missing data, such as patient demographics, clinical history and test results.

A complete list of outcomes can be found in our clinical trials registration and in the online supplemental material 1.

Primary outcomes are:

1. The sensitivity and specificity of the Gynocular, HRHPV and VIA when used as stand-alone tests to detect CIN2+ among WLHIV.

Secondary outcomes are:

1. Other test accuracy measures of the Gynocular, HRHPV testing and VIA which include positive and negative predictive values, positive and negative likelihood ratios, false-positive rate, false-negative rate, number needed to screen, and area under the receiver operating characteristic (ROC) curve and the diagnostic ORs.

2. Estimates of accuracy for tests when they are done in sequence. For example, HR-HPV positive test followed by Gynocular, and HR-HPV positive test followed by VIA.

3. Estimates of test accuracy in subgroups defined by age, menopausal status, parity, education, ART status, CD4 cell count, HIV RNA viral load, coinfection with $T$. vaginalis, methods of contraception, and earlier treatment for precancer and potential effect modification. The specific categorisation of the variables in the subgroup analysis is defined in the online supplemental material 2.

4. Area under the ROC curve for the Swede score determined by Gynocular.

A detailed statistical analysis plan has been drafted as a separate document, to describe planned analyses for the predefined outcomes and planned subgroup analyses. This can be found as an attachment to our clinical trials registration. In summary, two-by-two tables will be created to calculate sensitivity and specificity, positive and negative predictive values, positive and negative likelihood ratios, false-positive rate, false-negative rate, diagnostic odds ratio, and number needed to screen. Test accuracies will be calculated for each test in a stand-alone and add-on capacity, with corresponding confidence intervals using the Wilson score method. ${ }^{31}$ Area under the ROC curve for the Swede score as determined by the Gynocular will also be calculated. We will also evaluate test accuracy in subgroups defined by age, menopausal status, parity, education, ART status, CD4 cell count, HIV RNA viral load, coinfection with $T$. vaginalis, methods of contraception and earlier treatment for precancer. We will use logistic regression models to assess whether these patient characteristics have an influence on the association between each diagnostic test and disease status. 
Indeterminate results from the index test or reference standard will be reported, and sensitivity analysis will be performed to quantify the possible range of accuracy if participants with an indeterminate test or reference standard data are classified as positive or negative. The distribution of baseline characteristics between the participants with missing data and those without will be compared and managed similarly, with a sensitivity analysis to quantify the possible range of accuracy if participants with missing data are classified as diseased or non-diseased. If the missing data can be categorised as missing at random, such as lost samples, technical failures or accidental deviations from the protocol, multiple imputation may be used to reconstruct the data.

\section{Safety monitoring}

An independent Data Safety and Monitoring Board has been selected to safeguard the interests of study participants and advise principal investigators (PIs) on study validity and integrity.

\section{DISCUSSION}

The limitations of VIA for screening WLHIV have been widely noted, and the WHO acknowledges the need for improved screening strategies. ${ }^{32}$ HR-HPV testing is being promoted; however, in settings where it is not available, VIA is preferred over no screening. ${ }^{28}{ }^{32}$ In order to minimise loss to follow-up, same day screening and treatment is also recommended in many countries. Some HPV testing platforms have the capacity to process samples quickly and provide results after $60 \mathrm{~min}$, making a 'screen and treat' approach possible. We use an HPV testing platform that has been shortlisted by the WHO to run in this capacity. ${ }^{33}$ Our study fills an important evidence gap by exploring new strategies for the detection of precancerous cervical lesions in WLHIV in LMICs. Deriving estimates of test accuracy for all three tests in the same study population reduces bias and strengthens the internal validity of the study. Although studies show that HPV is more sensitive than VIA, specificity is relatively low, especially in WLHIV. ${ }^{34-36}$ Therefore, our study design allows the assessment of tests in both a stand-alone and add-on capacity. We also take measures to minimise two important forms of bias that compromise similar studies, including verification bias and misclassification bias.

Identifying true disease depends on the accurate placement of biopsies. A true gold standard would be to obtain a large loop excision of the transformation zone of every enrolled woman, but this approach would be unethical due to the complications of this procedure for women of childbearing age. ${ }^{37}$ In order to reduce misclassification, we use histopathology results of multiple punch biopsies obtained from the cervix, as the 'reference standard'. Several studies show that obtaining multiple biopsies improves detection of CIN2+ lesions compared with the practice of one biopsy from the most severe cervical lesion. Wentzensen et al found that sensitivities for detecting CIN2+ increased from $61 \%(95 \%$ CI $55 \%$ to $67 \%$ ) in a single biopsy, to $86 \%$ (95\% CI $80 \%$ to $90 \%$ ) with two biopsies, and $96 \%$ (95\% CI $91 \%$ to $99 \%$ ) with three biopsies. ${ }^{38}$ This was verified by the same research group ${ }^{39}$ and supported by other research groups in Asia and Europe. ${ }^{40-44}$ In addition, taking biopsies in the absence of abnormal colposcopic findings increases detection of high-grade lesions. ${ }^{35} 40$ 42-46 Many studies on colposcopic techniques include the use of multiple biopsies, even when lesions are not seen. ${ }^{8} 1638394244-52$ In our study, all acetowhite lesions are biopsied. When no lesion is seen, one biopsy is taken from each quadrant within the transformation zone. Furthermore, true disease is defined as the sum of all positive lesions identified at two close time points (baseline and 6 months), to ensure any lesions missed at baseline can be included. We chose a short follow-up period so we could identify CIN2+ cases that were missed at baseline. We expect this follow-up will pick up missed cases from baseline instead of progression or new cases. This may allow us to estimate true disease more accurately, but such a short follow-up cannot reliably be used to identify progression or new disease. However, this is not the objective of our study. We will include women who may have sexually transmitted infections (STIs), as this situation occurs in real life. Trichomoniasis is the most common STI in this setting. Because the inflammation caused by $T$. vaginalis may affect visual assessment of the cervix, we will assess test accuracy in women with and without coinfection, using GeneXpert for sensitive and specific detection of $T$. vaginalis. A limitation of this work is the small number of screening tests being evaluated. Other screening tests, including Papanicolaou testing, testing for DNA methylation and HPV oncoproteins E6 and E7, were considered, but these are more difficult to implement in our study setting.

To meet the 2030 WHO targets for the elimination of cervical cancer, new strategies are required to address pervasive problems in LMICs, such as transport for patients to hospitals and clinics, a lack of gynaecologists and other trained healthcare workers, and access to laboratory equipment. Urgent investigation into the best methods of screening for WLHIV are required, and we present a study investigating relevant tests that could be employed in screen and treat settings using methods to minimise bias. The results from this work may be useful in informing future screening guidelines for WLHIV in sub-Saharan Africa.

\section{Patient and public involvement}

Patients and members of the public were not involved in the design of this study. We invite verbal or written feedback from participants throughout the running of the study by facilitating direct communication study nurses or the site-principal investigator as required.

\section{Dissemination}

Results will be shared with patients and stakeholders. We will disseminate the findings of this study using a 
range of methods, including the use of academic media (peer-reviewed journal articles, national and international conference presentations), electronic and postal mail (posting of study findings to participants), community and stakeholder engagement activities (including provision of findings to the Cervical Cancer Prevention Programme and Ministry of Health in Zambia) and social media.

\section{Ethics}

This study received ethical approval from: National Health Research Authority and the University of Zambia Biomedical Research Ethics Committee (ref: 014-0918), the Zambia Medicines Regulatory Authority (ref: DMS/7/9/22/CT/084), the International Agency for Research on Cancer (IEC project number 18-15) and Swissethics (ref: 2018-01399). The protocol follows the 2015 STARD guidelines, as will the subsequent report of findings.

\section{Protocol and data availability}

The protocol, as approved by the respective ethical committees, is available on request to the corresponding author of this manuscript. Anonymised study data will be stored for ten years. We plan to publish results in open access journals with data available in accordance with 'FAIR principles'.

\section{Author affiliations}

${ }^{1}$ Institute of Social and Preventive Medicine (ISPM), University of Bern, Bern, Switzerland

${ }^{2}$ Graduate School for Cellular and Biomedical Sciences, University of Bern, Bern, Switzerland

${ }^{3}$ Centre for Infectious Disease Research in Zambia (CIDRZ), Lusaka, Zambia ${ }^{4}$ Obstetrics and Gynaecology, University Teaching Hospital, Lusaka, Zambia ${ }^{5}$ Women and Newborn health, Levy Mwanawasa Medical University Hospital, Lusaka, Zambia

${ }^{6}$ International Agency for Research on Cancer (IARC), World Health Organization, Lyon, France

${ }^{7}$ CTU Bern, University of Bern, Bern, Switzerland

${ }^{8}$ ARTORG Center for Biomedical Engineering Research, University of Bern, Bern, Switzerland

${ }^{9}$ University of Alabama at Birmingham (UAB), Birmingham, Alabama, USA

\section{Twitter Nicola Low @nicolamlow}

Acknowledgements We are grateful to Dr Srabani Mittal (in memoriam), who organised and participated in an on-site training course in Zambia during the preparatory phase of this study. We are also grateful to Professor Matthias Egger for his initial pivotal support, his guidance in grant applications and ongoing oversight for the study. We thank Jane Matambo for her support in obtaining national guideline documents, her assistance with setting up training courses, referring gynaecologists to join the study, piloting of data collection tools and general advice throughout implementation. We gratefully acknowledge Dr Steve Badman and Professor Andrew Vallely for sharing their experiences with the use of the GeneXpert device. Thanks to Dr Pierre Vassilakos, who provided guidance on histological assessment of cervical biopsies. Thanks, too, to our DSMB members: Professor Patrick Petignat (chair), Dr Sven Trelle, Dr Jake Pry, Dr Ketty Lubeya and Dr lacopo Baussano. We also thank Kali Tal for her contribution to editing this manuscript.

Contributors PB, MM, KT, JB, AL, AM, NL, TT and RS—overall concept and full protocol. JB, KT, AM, NL, MM and PB - grant applications. KT, MM, AM and $\mathrm{JB}$ - ethical and regulatory approvals. AM, MHM, MM, HK, KT, PB, JB, NL, KH and $\mathrm{SA}$-implementation of the study. AM, MHM, MM, HK and TT—coordination at the clinical site. All authors contributed to writing or editing the manuscript and approved its final form.

Funding This work was supported by Swiss Cancer Research, grant number KFS-4156-02-2017; National Institute of Allergy and Infectious Diseases of the National Institutes of Health, grant number U01AI069924; and ESTHER Switzerland foundation, grant number 171222 . This project has also received funding from the European Union's Horizon 2020 research and innovation programme under the Marie Skłodowska-Curie grant agreement No 801076, through the SSPH+ Global PhD Fellowship Programme in Public Health Sciences (GlobalP3HS) of the Swiss School of Public Health, and the Federal Commission for Scholarships for Foreign Students for the Swiss Government Excellence Scholarship (ESKAS No. 2019.0741) for the academic years 2019-2021.

Disclaimer Where authors are identified as personnel of the International Agency for Research on Cancer/World Health Organization, the authors alone are responsible for the views expressed in this article, and they do not necessarily represent the decisions, policy or views of the International Agency for Research on Cancer/World Health Organization.

Competing interests None declared.

Patient consent for publication Not required.

Provenance and peer review Not commissioned; externally peer reviewed.

Supplemental material This content has been supplied by the author(s). It has not been vetted by BMJ Publishing Group Limited (BMJ) and may not have been peer-reviewed. Any opinions or recommendations discussed are solely those of the author(s) and are not endorsed by BMJ. BMJ disclaims all liability and responsibility arising from any reliance placed on the content. Where the content includes any translated material, BMJ does not warrant the accuracy and reliability of the translations (including but not limited to local regulations, clinical guidelines, terminology, drug names and drug dosages), and is not responsible for any error and/or omissions arising from translation and adaptation or otherwise.

Open access This is an open access article distributed in accordance with the Creative Commons Attribution Non Commercial (CC BY-NC 4.0) license, which permits others to distribute, remix, adapt, build upon this work non-commercially, and license their derivative works on different terms, provided the original work is properly cited, appropriate credit is given, any changes made indicated, and the use is non-commercial. See: http://creativecommons.org/licenses/by-nc/4.0/.

\section{ORCID iDs}

Katayoun Taghavi http://orcid.org/0000-0003-0812-0069

Nicola Low http://orcid.org/0000-0003-4817-8986

\section{REFERENCES}

1 Bray F, Ferlay J, Soerjomataram I, et al. Global cancer statistics 2018: GLOBOCAN estimates of incidence and mortality worldwide for 36 cancers in 185 countries. CA Cancer J Clin 2018;68:394-424.

2 Ginsburg O, Bray F, Coleman MP, et al. The global burden of women's cancers: a grand challenge in global health. Lancet 2017;389:847-60.

3 Rohner E, Bütikofer L, Schmidlin K, et al. Cervical cancer risk in women living with HIV across four continents: a multicohort study. Int $J$ Cancer 2020;146:601-9.

4 Coleman JS, Cespedes MS, Cu-Uvin S, et al. An insight into cervical cancer screening and treatment capacity in sub Saharan Africa. $J$ Low Genit Tract Dis 2016:20:31-7.

5 Simms KT, Steinberg J, Caruana M, et al. Impact of scaled up human papillomavirus vaccination and cervical screening and the potential for global elimination of cervical cancer in 181 countries, 2020-99: a modelling study. Lancet Oncol 2019;20:394-407.

6 Viviano M, DeBeaudrap P, Tebeu P-M, et al. A review of screening strategies for cervical cancer in human immunodeficiency viruspositive women in sub-Saharan Africa. Int $J$ Womens Health 2017:9:69-79.

7 Bigoni J, Gundar M, Tebeu P-M, et al. Cervical cancer screening in sub-Saharan Africa: a randomized trial of via versus cytology for triage of HPV-positive women. Int J Cancer 2015;137:127-34.

8 Chibwesha CJ, Frett B, Katundu K, et al. Clinical performance validation of 4 point-of-care cervical cancer screening tests in HIVinfected women in Zambia. J Low Genit Tract Dis 2016;20:218-23.

9 Huchko MJ, Sneden J, Sawaya G, et al. Accuracy of visual inspection with acetic acid to detect cervical cancer precursors among HIV-infected women in Kenya. Int J Cancer 2015;136:392-8. 
10 Orang'o O, Liu T, Christoffersen-Deb A, et al. Use of via, Pap smear, or HR-HPV testing in women living with HIV/AIDS for post-treatment cervical cancer screening: same tests, different priorities. AIDS 2017;31:233-40.

11 Firnhaber C, Mao L, Levin S, et al. Evaluation of a cervicographybased program to ensure quality of visual inspection of the cervix in HIV-infected women in Johannesburg, South Africa. J Low Genit Tract Dis 2015;19:7-11.

12 World Health Organization (WHO). Draft: global strategy towards the elimination of cervical cancer as a public health problem Geneva, Switzerland, 2019. Available: https://www.who.int/publications/m/ item/draft-global-strategy-towards-eliminating-cervical-cancer-as-apublic-health-problem

13 Fokom-Domgue J, Combescure C, Fokom-Defo V, et al. Performance of alternative strategies for primary cervical cancer screening in subSaharan Africa: systematic review and meta-analysis of diagnostic test accuracy studies. BMJ 2015;351:h3084.

14 Sankaranarayanan R, Anorlu R, Sangwa-Lugoma G, et al. Infrastructure requirements for human papillomavirus vaccination and cervical cancer screening in sub-Saharan Africa. Vaccine 2013;31 Suppl 5:F47-52.

15 Clifford GM, Gonçalves MAG, Franceschi S, et al. Human papillomavirus types among women infected with HIV: a metaanalysis. AIDS 2006;20:2337-44.

16 Basu P, Banerjee D, Mittal S, et al. Evaluation of a compact, rechargeable, magnifying device to triage via and HPV positive women in a cervical cancer screening program in rural India. Cancer Causes Control 2016;27:1253-9.

17 Ngonzi J, Bajunirwe F, Wistrand C, et al. Agreement of colposcope and gynocular in assessment of cervical lesions by swede score: a randomized, crossover pilot trial. J Low Genit Tract Dis 2013;17:372-7.

18 Nessa A, Roy JS, Chowdhury MA, et al. Evaluation of the accuracy in detecting cervical lesions by nurses versus doctors using a stationary colposcope and Gynocular in a low-resource setting. BMJ Open 2014:4:e005313.

19 Nessa A, Wistrand C, Begum SA, et al. Evaluation of stationary colposcope and the Gynocular, by the swede score systematic colposcopic system in via positive women: a crossover randomized trial. Int J Gynecol Cancer 2014;24:339-45.

20 Kallner HK, Persson M, Thuresson M, et al. Diagnostic colposcopic accuracy by the GYNOCULAR and a stationary COLPOSCOPE. Int $J$ Technol Assess Health Care 2015;31:181-7.

21 Strander B, Ellström-Andersson A, Franzén S, et al. The performance of a new scoring system for colposcopy in detecting high-grade dysplasia in the uterine cervix. Acta Obstet Gynecol Scand 2005;84:1013-7.

$22 \mathrm{Hu}$ L, Bell D, Antani S, et al. An observational study of deep learning and automated evaluation of cervical images for cancer screening. $J$ Natl Cancer Inst 2019;111:343-4.

23 Crucitti T, Jespers V, Mulenga C, et al. Non-Sexual transmission of Trichomonas vaginalis in adolescent girls attending school in Ndola, Zambia. PLoS One 2011;6:e16310.

24 Torondel B, Sinha S, Mohanty JR, et al. Association between unhygienic menstrual management practices and prevalence of lower reproductive tract infections: a hospital-based cross-sectional study in Odisha, India. BMC Infect Dis 2018;18:473.

25 Sellors JW, Sankaranarayanan R. Colposcopy and treatment of cervical intraepithelial neoplasia: a beginners' manual. Lyon: IARC Press, 2003. https://screening.iarc.fr/doc/ Colposcopymanual.pdf

26 Ranga R, Rai S, Kumari A, et al. A comparison of the strength of association of Reid colposcopic index and swede score with cervical histology. J Low Genit Tract Dis 2017;21:55-8.

27 Bateman AC, Katundu K, Mwanahamuntu MH, et al. The burden of cervical pre-cancer and cancer in HIV positive women in Zambia: a modeling study. BMC Cancer 2015;15:541.

28 Parham GP, Sahasrabuddhe VV, Mwanahamuntu MH, et al. Prevalence and predictors of squamous intraepithelial lesions of the cervix in HIV-infected women in Lusaka, Zambia. Gynecol Oncol 2006;103:1017-22.

29 World Health Organization. Department of HIV/AIDS, guideline on when to start antiretroviral therapy and on pre-exposure prophylaxis for HIV, 2015. Available: https://www.who.int/hiv/pub/guidelines/ earlyrelease-arv/en
30 Hall MT, Smith MA, Simms KT, et al. The past, present and future impact of HIV prevention and control on HPV and cervical disease in Tanzania: a modelling study. PLoS One 2020;15:e0231388.

31 Wilson EB. Probable inference, the law of succession, and statistical inference. J Am Stat Assoc 1927;22:209-12.

32 World Health Organisation. WHO comprehensive cervical cancer control, 2014. Available: http://www.who.int/reproductivehealth/ publications/cancers/cervical-cancer-guide/en/

33 World Health Organisation. Who prequalification of in vitro diagnostics public report PQDx 0268-070-00 who PQ public report December 2017/version 3.0, 2017. Available: https://www.who.int/ diagnostics_laboratory/evaluations/pq-list/hiv-vrl/171221_final_pq_ report_pqdx_0268_070_00.pdf?ua=1

34 Chung MH, McKenzie KP, De VH, et al. Comparing Pap smear, via, and HPV cervical cancer screening methods among HIVpositive women by immune status and antiretroviral therapy. Aids 2013;27:2909-19.

35 Firnhaber C, Mayisela N, Mao L, et al. Validation of cervical cancer screening methods in HIV positive women from Johannesburg South Africa. PLoS One 2013;8:e53494-9.

36 Kremer WW, van Zummeren M, Breytenbach E, et al. The use of molecular markers for cervical screening of women living with HIV in South Africa. AIDS 2019;33:2035-42.

37 Jin G, LanLan Z, Li C, et al. Pregnancy outcome following loop electrosurgical excision procedure (LEEP) a systematic review and meta-analysis. Arch Gynecol Obstet 2014;289:85-99.

38 Wentzensen N, Walker JL, Gold MA, et al. Multiple biopsies and detection of cervical cancer precursors at colposcopy. J Clin Oncol 2015;33:83-9.

39 Wentzensen N, Walker J, Smith K, et al. A prospective study of risk-based colposcopy demonstrates improved detection of cervical precancers. Am J Obstet Gynecol 2018;218:604.e1-604.e8.

40 Gage JC, Hanson VW, Abbey K, et al. Number of cervical biopsies and sensitivity of colposcopy. Obstet Gynecol 2006;108:264-72.

41 van der Marel J, van Baars R, Rodriguez A, et al. The increased detection of cervical intraepithelial neoplasia when using a second biopsy at colposcopy. Gynecol Oncol 2014;135:201-7.

42 Hu S-Y, Zhang W-H, Li S-M, et al. Pooled analysis on the necessity of random 4-quadrant cervical biopsies and endocervical curettage in women with positive screening but negative colposcopy. Medicine 2017;96:e6689.

43 Zhao Y, Song Y, Zhao F, et al. [Value of 4-quadrant biopsies under colposcopy for detecting precancerous lesions in cervical cancer screening]. Zhonghua Zhong Liu Za Zhi 2015;37:875-9.

44 Baasland I, Hagen B, Vogt C, et al. Colposcopy and additive diagnostic value of biopsies from colposcopy-negative areas to detect cervical dysplasia. Acta Obstet Gynecol Scand 2016;95:1258-63.

45 Huh WK, Sideri M, Stoler M, et al. Relevance of random biopsy at the transformation zone when colposcopy is negative. Obstet Gynecol 2014;124:670-8.

46 Pretorius RG, Belinson JL, Azizi F, et al. Utility of random cervical biopsy and endocervical curettage in a low-risk population. J Low Genit Tract Dis 2012;16:333-8.

47 Pretorius RG, Zhang W-H, Belinson JL, et al. Colposcopically directed biopsy, random cervical biopsy, and endocervical curettage in the diagnosis of cervical intraepithelial neoplasia II or worse. Am J Obstet Gynecol 2004;191:430-4.

48 Bateman AC, Parham GP, Sahasrabuddhe VV, et al. Clinical performance of digital cervicography and cytology for cervical cancer screening in HIV-infected women in Lusaka, Zambia. J Acquir Immune Defic Syndr 2014;67:212-5.

49 Ghosh I, Mittal S, Banerjee D, et al. Study of accuracy of colposcopy in via and HPV detection-based cervical cancer screening program. Aust N Z J Obstet Gynaecol 2014;54:570-5.

50 Tebeu P-M, Fokom-Domgue J, Crofts V, et al. Effectiveness of a twostage strategy with HPV testing followed by visual inspection with acetic acid for cervical cancer screening in a low-income setting. Int $J$ Cancer 2015;136:E743-50.

51 Wang M, Hu S, Zhao S, et al. Accuracy of triage strategies for human papillomavirus DNA-positive women in low-resource settings: a cross-sectional study in China. Chin J Cancer Res 2017;29:496-509.

52 Sangrajrang S, Laowahutanont P, Wongsena M, et al. Comparative accuracy of Pap smear and HPV screening in Ubon Ratchathani in Thailand. Papillomavirus Res 2017;3:30-5. 